\title{
Binary quantum receiver concept demonstration
}

Chi-Wung Lau, Victor A. Vilnrotter, Sam Dolinar, JM Geremia, Hideo Mabuchi

Chi-Wung Lau, Victor A. Vilnrotter, Sam Dolinar, JM Geremia, Hideo Mabuchi, "Binary quantum receiver concept demonstration," Proc. SPIE 6105, Free-Space Laser Communication Technologies XVIII, 61050J (1 March 2006); doi: $10.1117 / 12.660268$

Event: Lasers and Applications in Science and Engineering, 2006, San Jose, California, United States 


\title{
Binary Quantum Receiver Concept Demonstration
}

\author{
Chi-Wung Lau, Victor A. Vilnrotter, Sam Dolinar, \\ Jet Propulsion Laboratory, California Institute of Technology \\ 4800 Oak Grove Dr., Pasadena, CA 91109-8099 \\ JM Geremia, Hideo Mabuchi \\ Physics and Control \& Dynamical Systems, California Institute of Technology \\ Norman Bridge Laboratory of Physics, MC 266-33 Pasadena, CA 91125
}

\begin{abstract}
An experimental demonstration of a quantum-optimal receiver for optical binary signals, developed as a joint effort by the Jet Propulsion Laboratory and the California Institute if Technology, is described in this article. A brief summary of the classical, quantum-optimal, and quantum near optimal solutions to detecting binary signals is first presented. The components and experimental setup used to implement the receivers is then discussed. Experimental performance and results for both optimal and near-optimal receivers are presented and compared to theoretical limits. Finally, experimental shortcomings are discussed along with possible solutions and future direction.
\end{abstract}

Keywords: quantum detection, Kennedy receiver, Dolinar receiver, quantum communications

\section{INTRODUCTION}

Quantum communications is a rapidly advancing field that potentially offers substantial gains to future deep space missions. As optical space communications become a reality, there will be a growing need for efficient receivers. The application of quantum communications to optical signals shows the greatest promise for deep-space quantum communications in the near future, since laser radiation characterized by coherent states propagates over vast distances suffering only attenuation, without altering its fundamental properties. This is in contrast to more exotic quantum states that rely on quantum entanglement, which quickly lose their uniquely quantum properties when substantial losses are introduced into the channel. Optical receivers contemplated for deep-space applications cannot improve significantly on existing RF communications system performance primarily because classical detection techniques and receiver structures do not approach the theoretical limits imposed by quantum mechanics. Current detection schemes involve detecting optical fields through energy detectors either directly or through the use of phase-sensitive coherent detection techniques. Even though quantum communications offer a significant advantage over classical receivers, it is not yet widely accepted due to the lack of known receiver structures which will perform the correct measurement to yield the quantum limit in performance. Known structures were believed to be too complex to implement in the real world and have been relegated to the realm of intellectual exercise.

With current advances in technology, quantum-optimal receivers can now be implemented and experimentally demonstrated. This article will demonstrate the initial attempt at building a quantum receiver for the case of binary signals. The purpose of this experiment was twofold. First, this experiment will show that quantum receivers can be built using the current level of technology. Second, this experiment will attempt to demonstrate the actual gains in performance of a quantum receiver. The case of binary signals was chosen since this is the only modulation in which the optimum quantum receiver structure is known. This optimum structure was devised and analyzed by Dolinar [1]. An

The research described in this publication was carried out by the Jet Propulsion Laboratory, California Institute of Technology, under contract with the National Aeronautics and Space Administration.

Free-Space Laser Communication Technologies XVIII, edited by G. Stephen Mecherle Proc. of SPIE Vol. 6105, 61050J, (2006) · 0277-786X/06/\$15 · doi: 10.1117/12.660268 
exponentially optimum (or "near optimum") receiver structure was devised earlier by Kennedy [2]. The experiment was performed as a two-step process. Rather than starting with an implementation of the complicated Dolinar receiver, the much less complex Kennedy receiver was first built and tested. After verifying the Kennedy performance, the Dolinar receiver was built and analyzed.

This article will begin by giving a brief overview of the performance of binary signals in both the classical and quantum regimes and show the gains possible by using an optimum quantum receiver over the classical counterpart in section 2 . In section 3, we will describe and show the experimental setup used to implement the Kennedy and Dolinar receivers. In section 4, we present the experimental results of the experiment. Section 5 contains a summary of this article. This experiment was performed at the California Institute of Technology under JM Geremia and Hideo Mabuchi.

\section{QUANTUM DETECTION OF BINARY SIGNALS}

We begin by defining the quantum mechanical description of binary signals. For binary signals, one of two hypotheses $\mathrm{H}_{0}$ and $\mathrm{H}_{1}$ are sent. These hypotheses will be represented by the two quantum state vectors $\left|\alpha_{0}\right\rangle$ and $\left|\alpha_{1}\right\rangle$ where each state vector represents a distinct coherent state. Coherent states can be expressed as a sum of number states $|n\rangle$ and have the form [3]:

$$
|\alpha\rangle=e^{-\frac{1}{2}|\alpha|^{2}} \sum_{n=0}^{\infty} \frac{\alpha^{n}}{(n !)^{1 / 2}}|n\rangle
$$

Coherent states are not orthogonal and the squared overlap between two distinct coherent states is:

$$
\left|\left\langle\alpha_{1} \mid \alpha_{2}\right\rangle\right|^{2}=\left|e^{-\left(\left|\alpha_{1}\right|^{2}+\left|\alpha_{2}\right|^{2}\right) / 2} \sum_{n} \sum_{m} \frac{\alpha_{1}^{n}}{\sqrt{n !}} \frac{\left(\alpha_{2}^{*}\right)^{m}}{\sqrt{m !}}\langle n \mid m\rangle\right|^{2}=\left|e^{-\left(\left|\alpha_{1}\right|^{2}+\left|\alpha_{2}\right|^{2}-2 \alpha_{1} \alpha_{2}^{*}\right) / 2}\right|^{2}=e^{-\left|\alpha_{1}-\alpha_{2}\right|^{2}}
$$

For binary signals, our modulations can take on the form of either On-Off Keying (OOK) or Binary Phase shift Keying (BPSK). The modulation scheme chosen for the experiment is BPSK. In BPSK, the two signal states can take on the form $\left|\alpha_{1}\right\rangle=|\alpha\rangle$ and $\left|\alpha_{2}\right\rangle=|-\alpha\rangle$ where the signal states are separated in phase space by $\pi$ and the average number of signal photons (averaged over both signals) is $K_{s}=|\alpha|^{2}$. For BPSK, the signal overlap is $\left|\left\langle\alpha_{1} \mid \alpha_{2}\right\rangle\right|^{2}=e^{-4 K_{s}}$. The classical scheme to detect these signals consists of adding a local field of great amplitude, in phase, to the received field and detecting the resulting sum field using classical energy detection in the absence of thermal noise. The probability of error performance of this coherent detector is [4]:

where

$$
\begin{aligned}
& P(E)=Q\left(\sqrt{4 K_{s}}\right) \\
& Q(x) \equiv \frac{1}{\sqrt{2 \pi}} \int_{x}^{\infty} e^{-y^{2} / 2} d y
\end{aligned}
$$

The optimum quantum mechanical receiver for detection of binary coherent states was devised and analyzed by Dolinar [1]. The Dolinar receiver requires adding a time-varying local field to the incoming signal field and performing photon counting on the combined field. The local field is controlled by a complex feedback loop in which both the amplitude and phase. The phase is varied based on the photon counts detected. The choice between hypotheses $\mathrm{H}_{0}$ and $\mathrm{H}_{1}$ for a time duration $\mathrm{T}$ is determined by the total counts $n$ observed. This yields the following decision rule [4]:

$$
\begin{array}{ll}
n \text { is } 0 \text { or even, } & \text { choose } \mathrm{H}_{1} \\
n \text { is odd, } & \text { choose } \mathrm{H}_{0}
\end{array}
$$


The Dolinar receiver achieves the Helstrom bound, yielding the error probability performance:

$$
P(E)=\frac{1}{2}\left[1-\sqrt{1-\left|\left\langle\alpha_{1} \mid \alpha_{2}\right\rangle\right|^{2}}\right]=\frac{1}{2}\left[1-\sqrt{1-e^{-4 K_{s}}}\right]_{K_{s} \rightarrow \infty} \cong \frac{1}{4} e^{-4 K_{s}}
$$

An exponentially optimum (or "near optimum") receiver structure was earlier devised by Kennedy [2]. The Kennedy architecture is physically much simpler than the Dolinar scheme due to the lack of the complex feedback loop. In this architecture, a local field of identical amplitude and phase of one of the possible signal fields is combined with the incoming signal field and photon counting is performed. The phase of this local field is set to null one hypothesis and is not varied unlike that of the Dolinar receiver. In this scheme, one hypothesis is always shifted to the ground state and the amplitude of the other hypothesis is always doubled. Assuming the local field nulls the $\mathrm{H}_{0}$ hypothesis, if no photons are detected, $\mathrm{H}_{0}$ is chosen while $\mathrm{H}_{1}$ is chosen in the event any counts are detected. In the absence of thermal noise, the only decision error occurs when the incoming field is doubled and no counts are observed. This yields the following decision rule [4]:

$$
\begin{array}{ll}
n \geq 1, & \text { choose } \mathrm{H}_{1} \\
n=0, & \text { choose } \mathrm{H}_{0}
\end{array}
$$

The average error probability performance is then:

$$
P(E)=\frac{1}{2} e^{-4 K_{s}}
$$

Performance curves for BPSK using both quantum and classical detection is shown in Fig. 1 [5]. It is interesting to note that quantum detection is exponentially $3 \mathrm{~dB}$ better in performance than coherent detection, practically achieving a 2.6 $\mathrm{dB}$ reduction in the required signal energy at an error probability of $10^{-5}$.

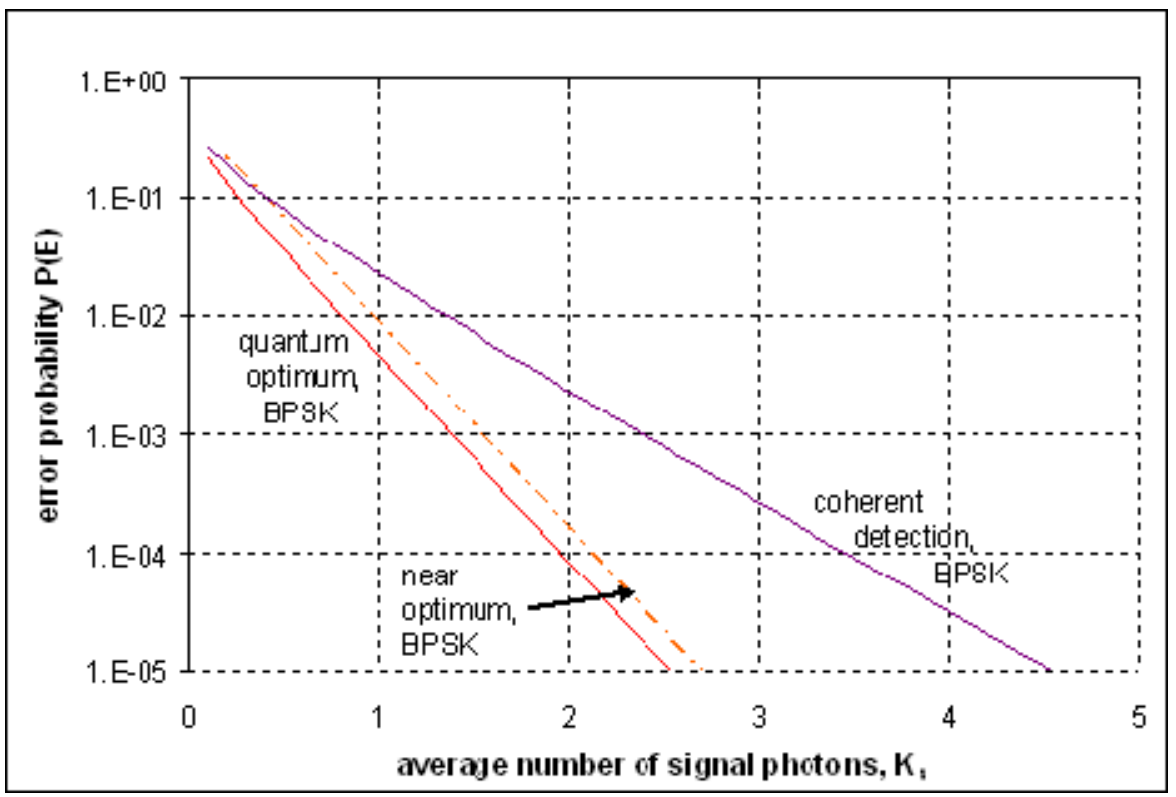

Figure 1: Performance of binary signals, no background radiation

Both the Kennedy and Dolinar receivers offer a clear advantage over classical detection. The question is how can these two architectures be implemented? 


\section{EXPERIMENTAL SETUP}

The experimental setup used to demonstrate the Kennedy and Dolinar receivers is depicted in Fig. 2.

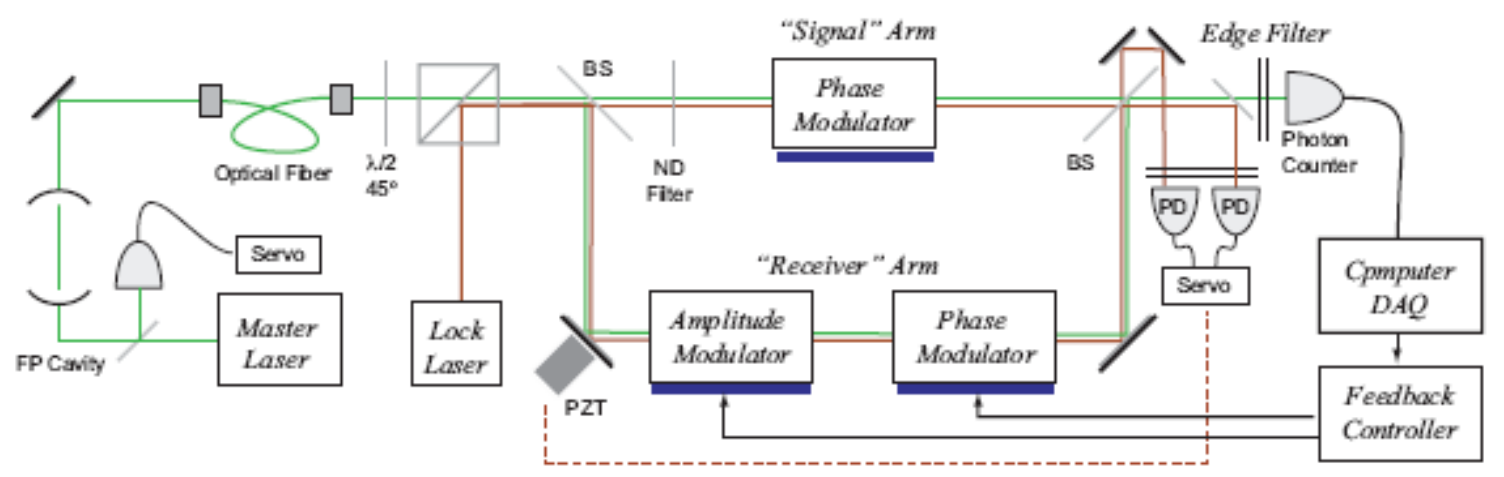

Figure 2: Block diagram of the experimental setup for the Kennedy and Dolinar receiver.

The setup has three primary significant components. The first component is the optical source. The optical source is provided by an external grating-stabilized tunable diode laser operating at $852 \mathrm{~nm}$ which is locked to a high-finesse optical cavity and then transmitted through a single-mode polarization maintaining optical fiber. The second component in the experiment is constructed as a pathlength-stabilized Mach-Zender interferometer. From Fig. 2, the top arm acts as the incoming signal beam and the lower arm acts as the receiver's LO. The top arm "signal" contains a phase modulator to switch between the binary phase-shifted coherent state signals. The lower "receiver" arm contains a phase and amplitude modulator. The lower arm also acts as the local oscillator in both the Dolinar and Kennedy receiver. The third component is the combined signal from both arms of the interferometer directed onto a single photon counting module whose output is processed by a computer and feedback control electronics. The feedback signal is used to drive the amplitude and phase modulators in the lower "receiver" arm of the interferometer.

The high-finesse cavity is used to filter classical intensity fluctuations, above that of shotnoise, from the optical source which provides both the signal and local oscillator. Shotnoise limited light is necessary in order to observe the intrinsic Poisson number fluctuations of an optical coherent state. The $L=20 \mathrm{~cm}$ Fabry-Perot cavity exhibited a finesse of $F \sim$ 75,000 and a linewidth of $5 \mathrm{kHz}$. Filtering the light through a single-mode fiber enables good free-space visibility for the interferometer, exceeding $99 \%$, limited only by the quality of the mode-matching. The interferometer pathlength had to be stabilized and this was accomplished through the use of a secondary laser ( $\mathrm{HeNe}$ at $633 \mathrm{~nm}$ ). The secondary laser generated a homodyne error signal which was then fed back to the piezoelectric modulators which controlled the optical pathlength. Phase modulation of both "signal" and "receiver" arms were performed using broadband high-voltage freespace coupled electro-optic modulators. The local oscillator intensity was controlled by a second, nested stabilized Mach-Zender interferometer with a broadband phase modulator in one arm.

The two "signal" and "receiver" arms were combined on the outcoupling beam-splitter of the interferometer and a single-photon counting module (Perkin-Elmer 100 dark counts / second) was used to monitor the combined signal. The output of the photon counter was recorded by a computer which also provided the necessary feedback electronics. The output voltage used to control the local oscillator intensity as well as for flipping the local oscillator phase was generated by a high-speed loop running on a National Instrument PCI-MIO16E4 data acquisition board. The feedback loop was also responsible for gating the photon counter at the beginning of each measurement period.

Fig. 3 is a photo of the experimental setup implementing the block diagram in Fig. 2. 


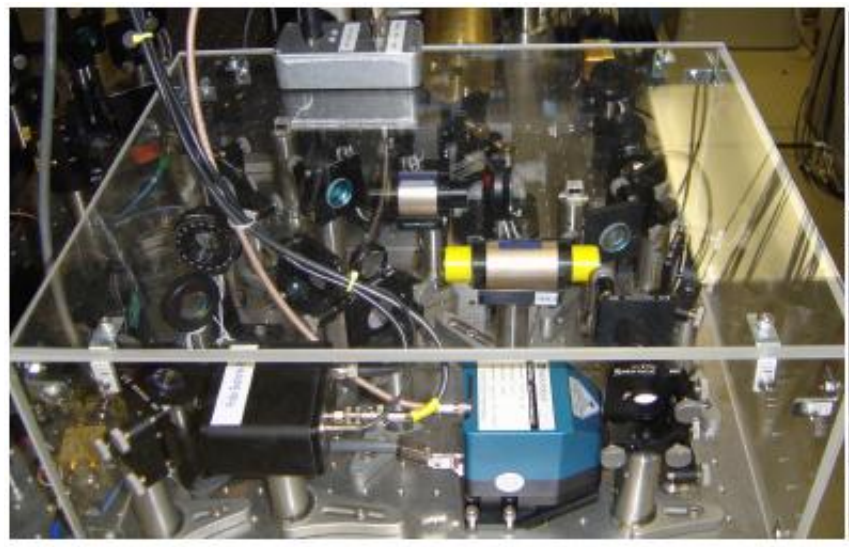

Figure 3: Photographs of the experimental setup: a) Laser

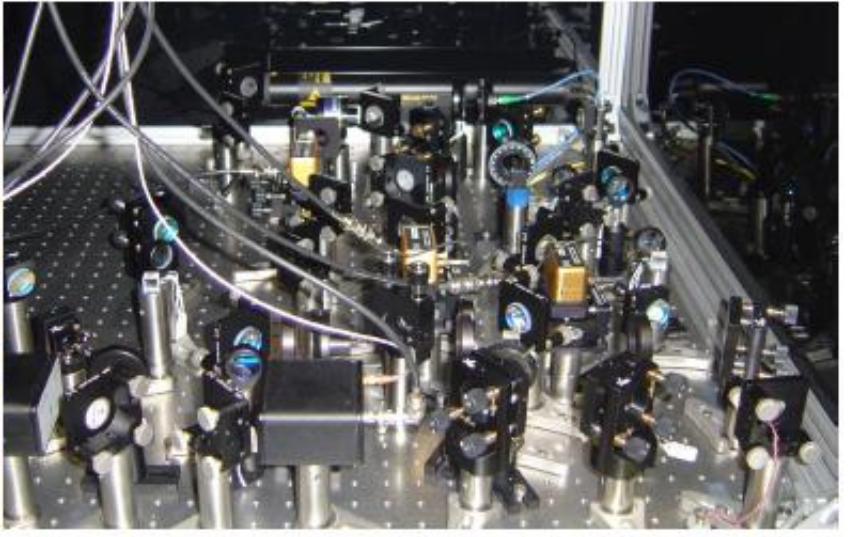

b) Interferometer

Fig. 3a shows the cavity-stabilized diode laser (blue laser head) operating at $852 \mathrm{~nm}$. The laser was locked to the cavity (brass cylinder at the top) using the standard Pound-Drever-Hall procedure and required a servo bandwidth of approximately $5 \mathrm{MHz}$. The other optical components in the photo were used for beam-shaping and optical isolation. Fig. $3 \mathrm{~b}$ shows the Mach-Zender interferometer, lock laser (black cylinder), and optical modulators (gold components) used to implement the Dolinar receiver.

\section{EXPERIMENTAL RESULTS}

The performance of the experimental setup for both the Kennedy and Dolinar receivers is shown in Fig. 4.
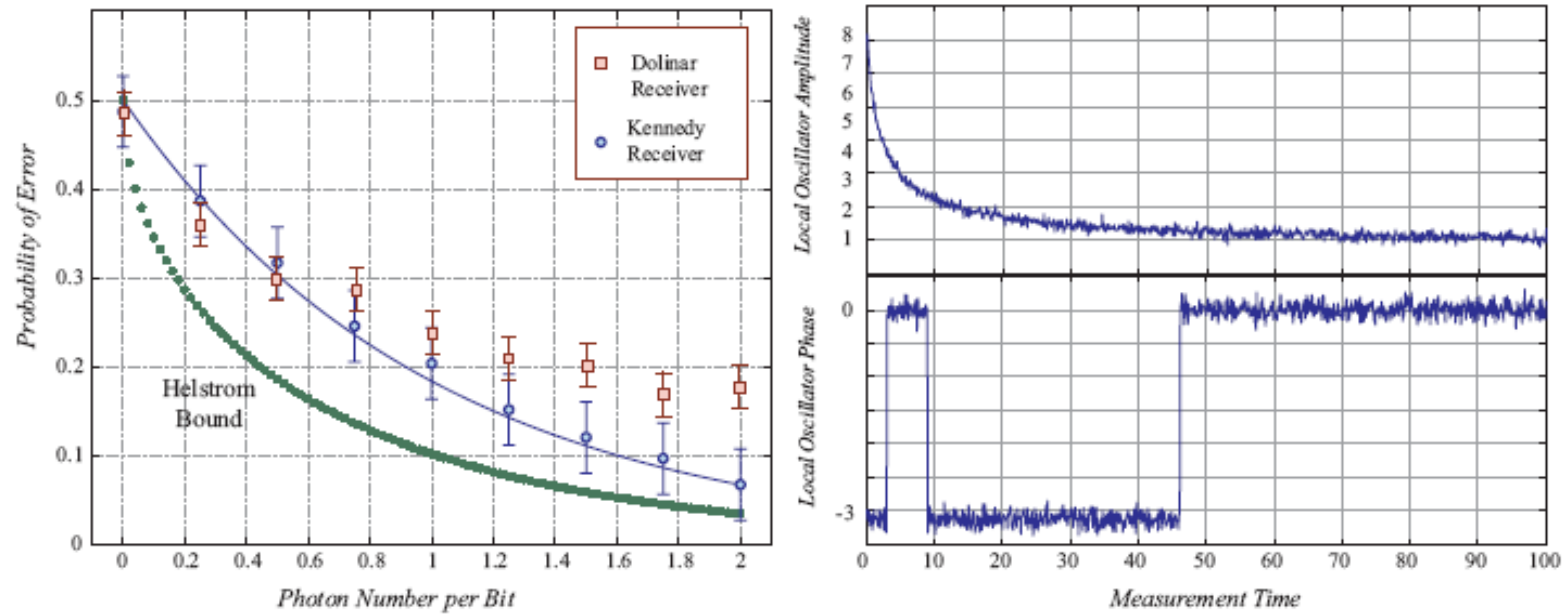

Figure 4: Measured performance of: a) Kennedy and Dolinar receivers, b) Dolinar receiver measurement trajectory.

The probability of error of the Kennedy and Dolinar receivers is shown in Fig. 4a. The performance was determined from 10,000 random bits with $p_{0}=p_{1}=1 / 2$, for both the Kennedy receiver (local oscillator set to cancel $\left|\alpha_{0}\right\rangle$ and with feedback disabled) and the initial attempt at the Dolinar receiver. We see that the Kennedy receiver data (blue circles) essentially achieves the theoretical shotnoise limit (blue solid line). However, this initial attempt at experimentally designing the Dolinar receiver, which should achieve the Helstrom bound, is seen to underperform the Kennedy receiver for all but the smallest mean photon numbers.

Fig. 4b depicts an example of the Dolinar receiver feedback trajectory. As can be seen, the amplitude of the local oscillator decreases from a maximum value, (ideally $\infty$ ), at the onset of the measurement and eventually settles down to 
be equal to the amplitude of the incoming signal. The profile of this signal is in fact deterministic and does not depend on the arrival of photons. In contrast, the local oscillator phase toggles between 0 and $\pi$ at each click from the photon counter. We see that three such events occur in this plot, and as expected, the apparent decrease in photon rate as the receiver gradually improves the degree to which it cancels the incoming signal.

From the plotted Dolinar receiver trajectory, we see that the feedback switching of the local oscillator occurs as expected in the experiment. However, the statistical performance is not as expected it should be and this prompts us to analyze why. As always with feedback control experiments, one should expect that delays in the application of the feedback actuation might be a problem. However, repeating the experimental data at lower bandwidth did not improve the performance, implying that delays are not the limiting factor. Rather, it appears that it is the accuracy with which the amplitude profile can be applied to the local oscillator that has prevented the receiver from achieving the optimum quantum bound. Limitations in this signal are due primarily to the limited visibility of the amplitude modulator, which is approximately 1:50 at this bandwidth.

\section{CONCLUSIONS}

Based on the results obtained in this initial demonstration, we see that the implementation of the Kennedy receiver matches that of the theoretical performance. The Dolinar receiver however, requires necessary improvements in order to match the Helstrom bound. The data indicate the major observed shortcoming appears to be the degree of intensity control. It is believed that the degree of intensity control must be improved from 1:50 to 1:1000. For the purposes of the experiment, amplitude modulators constructed from a NewFocus broadband electro-optic phase modulator (Model 4001) operated inside of a Mach-Zender interferometer and combined with prism polarizers) were used. In order to improve the intensity control to the ratio needed for the receiver to approach the quantum bound, we must use fiber-integrated modulators, such as those available from JDS Uniphase. These modulators are capable of operating at high bandwidth with very high mode matching $(>1: 1,000)$ and low control programming voltages. As an additional experiment for future work, a third receiver architecture could be implemented. This third architecture would be a Kennedy-Dolinar hybrid where the Kennedy receiver is used for the initial $\Delta$ time and the Dolinar receiver is used for the remaining time interval. The purpose of this third receiver would be to yield additional insight into the cause of the performance degradation observed in the Dolinar receiver and verify whether the amplitude profile is indeed the culprit. Additional work would also be to separate the signal and receiver lasers in order to more accurately model real world systems. This adds the complication that the signal and receiver lasers would no longer be precisely in phase necessitating the need for some phase tracking module.

This goal of this initial experiment was to show that receiver performance close to and at the quantum limit could indeed be achieved using the current level of technology. The Kennedy receiver was successfully implemented with performance matching theory. The initial attempt at the more complicated Dolinar receiver however, did not yield the expected performance. Despite the degradation in performance, the data suggests that the complications limiting the Dolinar receiver performance can be overcome with better amplitude modulators. This experiment has shown that receivers which perform near the quantum limit are realizable. The research conducted in this DRDF effort helps to

clarify and demonstrate the fundamental limits of quantum communications, leading to potential enhancement of JPL's deep-space communications capabilities.

\section{REFERENCES}

1. S. J. Dolinar, Jr. "An Optimum Receiver for the Binary Coherent State Quantum Channel," MIT Research Laboratory of Electronics Quarterly Progress Report 111, Massachussetts Institute of Technology, Cambridge, Massachussetts, pp. 115-120, October 15, 1973.

2. R. S. Kennedy, "A Near-Optimum Receiver for the Binary Coherent State Quantum Channel," MIT Research Laboratory of Electronics Quarterly Progress Report 108, Massachusetts Institute of Technology, Cambridge, Mass., pp. 219-225, January 15, 1973. 
3. R. J. Glauber, "Coherent and Incoherent States of the Radiation Field," The Physical Review, vol. 131, no. 6, pp. 2766-2788, 1963.

4. Carl W. Helstrom, Quantum Detection and Estimation Theory, Mathematics in Science and Engineering, Volume 123, Academic Press, New York, 1976.

5. V. Vilnrotter and C.-W. Lau, "Quantum Detection Theory for the Free-Space Channel," IPN Progress Report 42-146, Jet Propulsion Laboratory, August 15, 2001. 